\title{
A Rare Case of Primary Tubular Adenocarcinoma of the Thymus, Enteric Immunophenotype: A Case Study and Review of the Literature
}

Hae Yoen Jung · Hyundeuk Cho Jin-Haeng Chung ${ }^{1}$. Sang Byoung Bae ${ }^{2}$ Ji-Hye Lee $\cdot$ Hyun Ju Lee Si-Hyong Jang $\cdot$ Mee-Hye Oh

Department of Pathology, Soonchunhyang University Cheonan Hospital, Cheonan; ${ }^{1}$ Department of Pathology and Respiratory Center, Seoul National University Bundang Hospital, Seongnam; 'Division of Hematology and Oncology, Department of Internal Medicine, Soonchunhyang University Cheonan Hospital, Cheonan, Korea

Received: March 10, 2015

Revised: April 8, 2015

Accepted: April 16, 2015

\section{Corresponding Author}

Mee-Hye Oh, MD, PhD

Department of Pathology, Soonchunhyang

University Cheonan Hospital, 31 Suncheonhyang

6-gil, Dongnam-gu, Cheonan 330-930, Korea

Tel: $+82-41-570-3580$

Fax: $+82-41-570-3580$

E-mail: mhoh0212@schmc.ac.kr
Thymic carcinomas are uncommon malignant tumors, and thymic adenocarcinomas are extremely rare. Here, we describe a case of primary thymic adenocarcinoma in a 59-year-old woman. Histological examination of the tumor revealed tubular morphology with expression of cytokeratin 20 and caudal-type homeobox 2 according to immunohistochemistry, suggesting enteric features. Extensive clinical and radiological studies excluded the possibility of an extrathymic primary tumor. A review of the literature revealed only two global cases of primary tubular adenocarcinomas of the thymus with enteric immunophenotype.

Key Words: Thymus gland; Adenocarcinoma; Keratin-20; Caudal type homeobox 2
Thymic carcinomas are uncommon malignant tumors. According to the World Health Organization (WHO), thymic carcinomas are mostly squamous cell, lymphoepithelioma-like, or basaloid carcinomas. ${ }^{1}$ Thymic adenocarcinomas are extraordinarily rare. We herein report a case of primary thymic adenocarcinoma with tubular morphology and enteric immunophenotype as determined by expression of cytokeratin (CK) 20 and caudal type homeobox 2 (CDX2). The immunophenotype suggests metastasis from the gastrointestinal tract; however, extensive clinical evaluation revealed no extrathymic primary tumor. In addition, we review the literature to facilitate a discussion of the clinicopathologic characteristics of thymic adenocarcinoma.

\section{CASE REPORT}

A previously healthy 59-year-old woman presented with ab- dominal pain in the lower left quadrant that had persisted for 3 months. Endoscopic studies failed to reveal specific changes in the whole gastrointestinal tract mucosa. Imaging studies revealed an anterior mediastinal mass (Fig. 1A) and multiple lung nodules. Additionally, a whole-body positron emission tomography scan showed abnormal hyperuptake lesions in the left 10th rib and 10th vertebral body, suggesting metastasis (Fig. 1B). Laboratory tests indicated elevated levels of several serum tumor markers (carbohydrate antigen [CA] 19-9, $252.2 \mathrm{U} / \mathrm{mL} ; \mathrm{CA} 125$, $62.9 \mathrm{IU} / \mathrm{mL}$; and carcinoembryonic antigen, $8.73 \mathrm{ng} / \mathrm{mL}$ ).

The surgeon prioritized surgery for this patient not only because of her aggravated symptoms, but also because the tumor had invaded the left brachiocephalic vein. Approached through a median sternotomy, an extended thymectomy and combined resection of the pericardium were performed. Incomplete resection was inevitable because of tumor invasion into the pericar- 
dium, phrenic nerve, innominate vein, and aorta.

Gross examination identified a solid mass measuring $6.8 \times 4.7$ $\times 3.9 \mathrm{~cm}$. The tumor was unencapsulated and invaded the pericardium. The mass was firm and rubbery with a homogeneous gray-whitish cut surface. There was no internal fibrous septation (Fig. 2A). Microscopic examination revealed that the tumor was surrounded by normal thymic tissue and was composed of various-sized glandular structures (Fig. 2B). The tumor cells were columnar and oval, forming cystic, tubular, and cribriform structures containing necrotic material. Immunohistochemical staining showed focally positive $\mathrm{CK} 7$ staining and diffusely strong positive staining for $\mathrm{CK} 20$ and $\mathrm{CDX} 2$ (Fig. 2C-G) but negative staining for thyroid transcription factor 1 (TTF-1) and Napsin A. CD5 staining was positive for both tumor cells and T lymphocytes in the normal thymus parenchyma and stroma between tumor cell nests (Fig. 2H).

Clinically, the possibility of metastatic adenocarcinoma from the lung was raised; however, the multiple small lung nodules measured less than $1 \mathrm{~cm}$, and the tumor cells were negative for TTF-1 and Napsin A. Furthermore, the pathologic features suggested metastatic adenocarcinoma of enteric origin; however, physical and radiologic examinations did not reveal primary tumors elsewhere, including in the gastrointestinal tract. These observations collectively led to a pathologic diagnosis of primary thymic adenocarcinoma of enteric immunophenotype with tubular morphology. Adjunctively, extensive sampling revealed no mucin pools or papillary structures and no other histologic components such as thymic cysts, thymomas, or teratomas.

After the operation, palliative chemoradiotherapy and radiotherapy were administered. The patient is alive with aggravated bone and lung metastasis after 11 months of follow-up.

\section{DISCUSSION}

Primary thymic adenocarcinoma is very rare. It was first reported in 1989 by Moriyama et al. ${ }^{2}$ but was not accepted as a valid histologic subtype until 1997. ${ }^{3}$ The current WHO classification system subtypes thymic adenocarcinomas as either papillary or nonpapillary. Nonpapillary adenocarcinomas include a heterogeneous group of tumors, such as mucinous adenocarcinoma, adenocarcinoma with glandular differentiation, adenocarcinoma with adenoid cystic carcinoma features, and hepatoid carcinoma. ${ }^{1}$ This heterogeneity, coupled with the rarity of the carcinoma, has led to a paucity of information on the specific clinicopathologic features of thymic adenocarcinoma.

According to a series of case reports in which descriptive classifications are commonly used, thymic adenocarcinomas comprise papillary, ${ }^{4-6}$ mucinous, ${ }^{3,7-9}$ glandular (or tubular), ${ }^{10-12}$ and papillotubular ${ }^{2,13,14}$ adenocarcinomas. In 2003, Maghbool et al. ${ }^{3}$ reviewed 26 previous cases of reported thymic adenocarcinomas and demonstrated that the mucinous type shows a significantly worse prognosis than the papillary type. Moser et al. ${ }^{12}$ recently reported two cases of primary thymic adenocarcinoma with CK20 and CDX2 expression, which reflects enteric differentiation, and suggested this is a novel subtype along with 11 reported thymic adenocarcinomas with enteric immunophenotype.

For this study, we also reviewed English literature of thymic adenocarcinomas, except for thymic carcinomas with adenoid cystic carcinoma features. In analysis of clinicopathologic features, including the current case, there are currently 39 reports of primary thymic adenocarcinomas, consisting of 16 mucinous adenocarcinomas (41.0\%), 13 papillary adenocarcinomas (33.3\%), seven tubular adenocarcinomas (17.9\%), and three papillotubu-
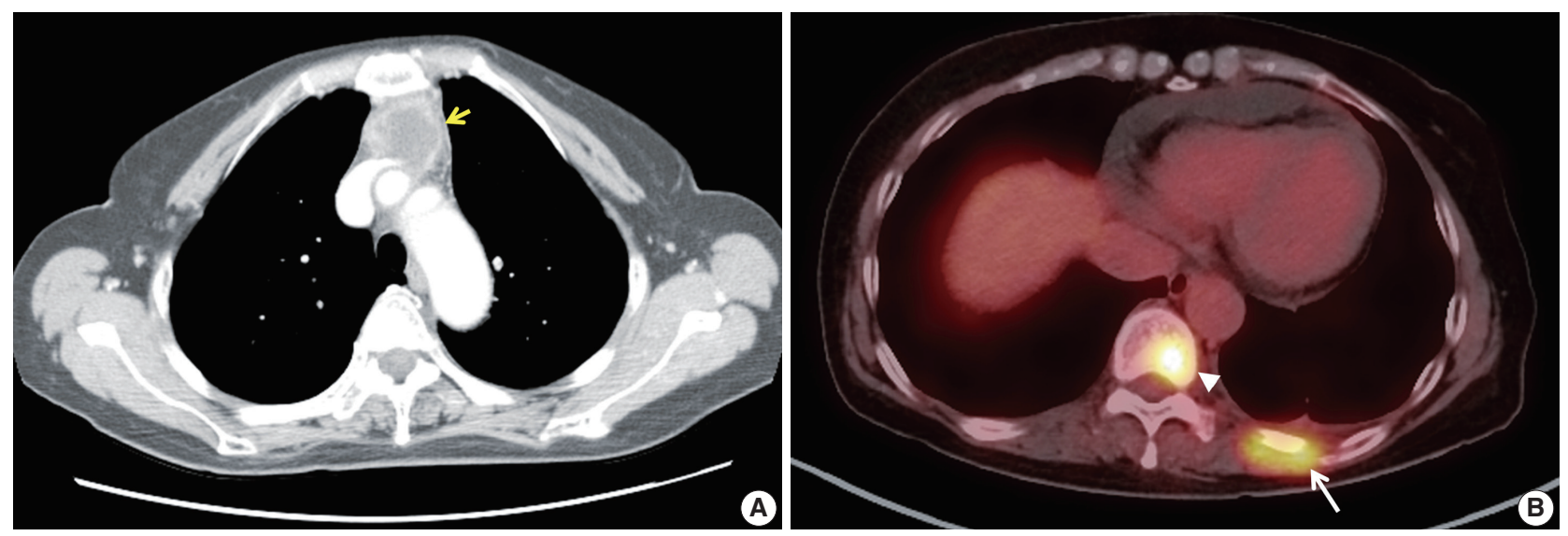

Fig. 1. Radiologic examination of the anterior mediastinum. (A) An irregularly enhancing mass (arrow) in the anterior mediastinum on a chest computed tomography scan. (B) Abnormal hyperuptake in lesions at the 10th vertebra (arrowhead) and the left 10th rib (arrow) on a wholebody positron emission tomography scan. 
lar adenocarcinomas (7.7\%). Among these cases, information on CK20 and/or CDX2 expression was available for 20 cases, revealing that 15 were enteric type and five were not.

The morphology of these enteric-type adenocarcinomas was mucinous in 11 cases, tubular in three cases, and papillotubular in one case. Furthermore, the enteric-type adenocarcinomas and mucinous/tubular adenocarcinomas have similar clinicopathologic features. A total of seven of the 14 cases of mucinous/tubular adenocarcinomas were accompanied by thymic cysts. Moreover, mucinous/tubular adenocarcinomas showed CD5 expression
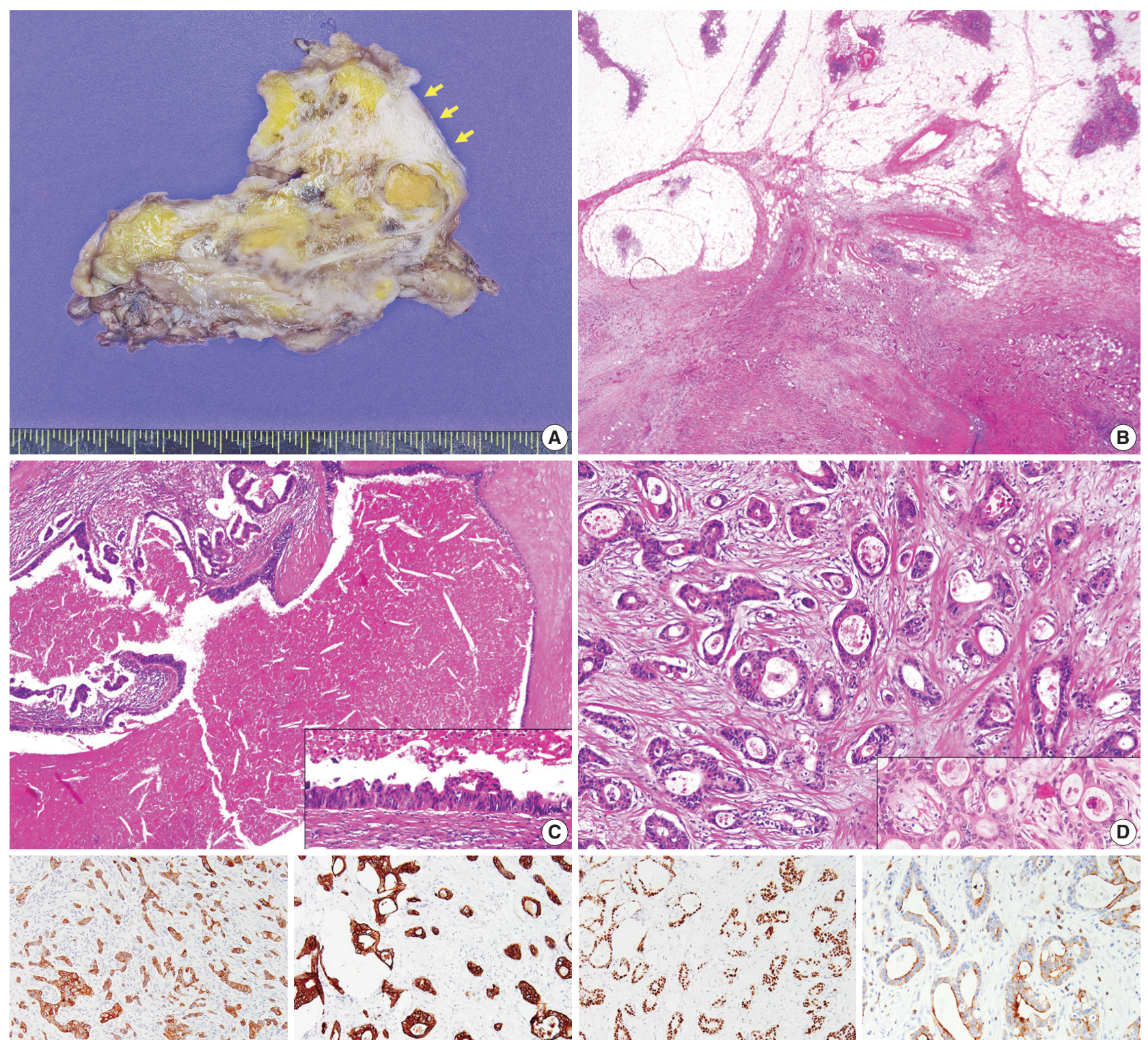

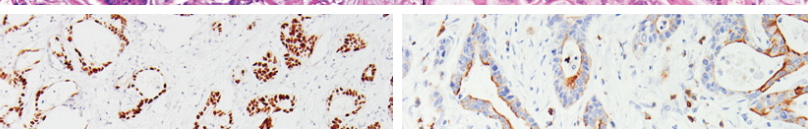

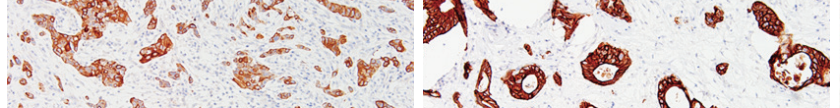

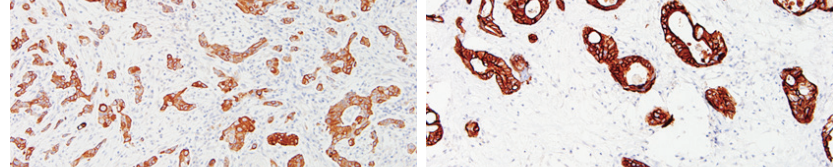
$0,-\infty$

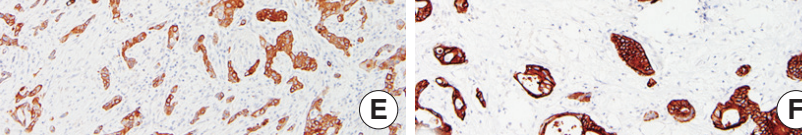
$4)^{2}+4$ a $20^{2} 3$ ?
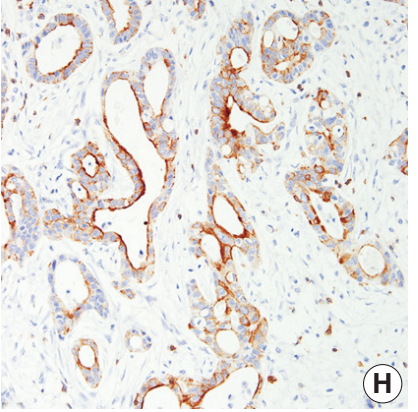

Fig. 2. Pathologic examination. (A) Gross examination of the tumor reveals an ill-defined mass with pericardial invasion (arrows, pericardium). (B) Microscopically, the tumor is surrounded by normal thymic tissues. At higher magnification, the tumor is composed of glandular or tubular structures with large glands lined by tall columnar cells (C) and oval cells forming small glands (D). The tumor cells show positive staining for CK7 (E), CK20 (F), CDX2 (G), and CD5 (H). CK, cytokeratin; CDX2, caudal type homeobox 2. 
more frequently $(76.4 \%)$ than papillary adenocarcinomas (33.3\%). Similarly, 12 of 15 enteric-type adenocarcinomas (80\%) showed CD5 expression, and eight (53.3\%) were accompanied by thymic cysts.

In general, low-grade histology (well-differentiated squamous cell carcinoma, mucoepidermoid carcinoma, and basaloid carcinoma) ${ }^{15}$ low Masaoka stage, and complete resection are factors known to be associated with better survival from thymic carcinoma. ${ }^{16,17}$ Kaplan-Meier analysis of thymic adenocarcinomas revealed that modified Masaoka stage I or II disease had better disease-free survival than stage III or IV disease $(\mathrm{p}=.040)$. Additionally, we found that mucinous/tubular subtype showed a trend toward poorer overall survival than papillary or papillotubular subtype, in line with previous findings, ${ }^{3}$ however, this result failed to reach statistical significance in Kaplan-Meier analysis $(\mathrm{p}=.610)$. Interestingly, the nonenteric type seemed to have worse prognosis than the enteric type (mean survival time, nonenteric vs enteric type, $22.1 \pm 7.4$ months vs $85.6 \pm 23.1$ months), especially for those with mucinous morphology (mean survival time, nonenteric vs enteric type, $7.3 \pm 4.2$ months vs $91.9 \pm 24.4$ months); however, the number of cases was too small to analyze for statistical significance. Other factors of sex, tumor size, associated thymic cyst or thymomas, serum tumor marker elevation, or c-kit or CD5 immunoexpression were not related to prognosis.

In summary, we report a rare case of primary tubular adenocarcinoma of the thymus with enteric immunophenotype. To the best of our knowledge, there have been only two such cases worldwide. The tumor presented at an advanced stage and demonstrated aggressive behavior. We believe that identification of more cases is essential in order to investigate the clinicopathologic characteristics of primary thymic adenocarcinomas.

\section{Conflicts of Interest}

No potential conflict of interest relevant to this article was reported.

\section{REFERENCES}

1. Müller-Hermelink HK, Marx A, Kuo TT, Kurrer M, Chen G, Shimosato Y. Non-papillary adenocarcinomas. In: Travis WD, Brambilla E, Müller-Hermelink HK, Harris CC, eds. World Health Organization classification of tumours: pathology and genetics of tumours of the lung, pleura, thymus and heart. 3rd ed. Lyon: IARC Press, $2004 ; 184$.

2. Moriyama S, Shimizu N, Kurita A, Teramoto S, Taguchi K. A case of adenocarcinoma of the thymus. Nihon Kyobu Geka Gakkai Zasshi
1989; 37: 717-22

3. Maghbool M, Ramzi M, Nagel I, et al. Primary adenocarcinoma of the thymus: an immunohistochemical and molecular study with review of the literature. BMC Clin Pathol 2013; 13: 17.

4. Matsuno Y, Morozumi N, Hirohashi S, Shimosato Y, Rosai J. Papillary carcinoma of the thymus: report of four cases of a new microscopic type of thymic carcinoma. Am J Surg Pathol 1998; 22: 873-80.

5. Yoshino M, Hiroshima K, Motohashi S, et al. Papillary carcinoma of the thymus gland. Ann Thorac Surg 2005; 80: 741-2.

6. Furtado A, Nogueira R, Ferreira D, Tente D, Eisele R, Parente B. Papillary adenocarcinoma of the thymus: case report and review of the literature. Int J Surg Pathol 2010; 18: 530-3.

7. Choi WW, Lui YH, Lau WH, Crowley P, Khan A, Chan JK. Adenocarcinoma of the thymus: report of two cases, including a previously undescribed mucinous subtype. Am J Surg Pathol 2003; 27: 124-30.

8. Ra SH, Fishbein MC, Baruch-Oren T, et al. Mucinous adenocarcinomas of the thymus: report of 2 cases and review of the literature. Am J Surg Pathol 2007; 31: 1330-6.

9. Abdul-Ghafar J, Yong SJ, Kwon W, Park IH, Jung SH. Primary thymic mucinous adenocarcinoma: a case report. Korean J Pathol 2012; 46: 377-81.

10. Sawai T, Inoue $Y$, Doi $S$, et al. Tubular adenocarcinoma of the thymus: case report and review of the literature. Int J Surg Pathol 2006; 14: 243-6.

11. Misao T, Yamamoto Y, Nakano H, Toyooka S, Yamane M, Satoh K. Primary thymic adenocarcinoma with production of carbohydrate antigen 19-9 and carcinoembryonic antigen. Jpn J Thorac Cardiovasc Surg 2004; 52: 30-2.

12. Moser B, Schiefer AI, Janik S, et al. Adenocarcinoma of the thymus, enteric type: report of 2 cases, and proposal for a novel subtype of thymic carcinoma. Am J Surg Pathol 2015; 39: 541-8.

13. Ishiwata T, Sekiya M, Suzuki T, Matsuoka T, Kumasaka T, Takahashi K. Thymic adenocarcinoma with sarcomatoid features characterized by intracaval tumor growth: report of a case. Surg Today 2010; 40: 1068-72.

14. Teramoto K, Kawaguchi Y, Hori T, et al. Thymic papillo-tubular adenocarcinoma containing a cyst: report of a case. Surg Today 2012; 42: 988-91.

15. Suster S, Rosai J. Thymic carcinoma: a clinicopathologic study of 60 cases. Cancer 1991; 67: 1025-32.

16. Wang S, Wang Z, Liu X, Wang D, Liu F. Prognostic factors of patients with thymic carcinoma after surgery: a retrospective analysis of 58 cases. World J Surg 2014; 38: 2032-8.

17. Filosso PL, Guerrera F, Rendina AE, et al. Outcome of surgically resected thymic carcinoma: a multicenter experience. Lung Cancer 2014; 83: 205-10. 